\title{
STICHWORT
}

\section{Unterstützte Kommunikation (UK)}

\author{
Susanne Wachsmuth
}

Kleine Kinder mit einer Behinderung haben häufig eine verzögerte Lautsprachentwicklung, und man kann noch nicht vorhersagen, wann diese einsetzen wird und ob die Kinder später über eine lautsprachliche Kompetenz verfügen werden, die es ihnen ermöglicht, alles zu verstehen und zu sagen, was ihnen wichtig ist. Die meisten Kinder erlangen die Lautsprache. Von einigen Kindern wird ein gutes Lautsprachverständnis erreicht, sie sind aber selber nicht in der Lage, hinreichend genau zu artikulieren, sodass sie gar nicht oder nur von vertrauten Menschen verstanden werden. Bei anderen Kindern ist die Lautsprachentwicklung so stark verzögert, dass sie Jahre später als sich regulär entwickelnde Kinder verstehen und sprechen können. Schließlich gibt es die Gruppe der Kinder, für die Zeit ihres Lebens die Lautsprache ein zu flüchtiges und zu komplexes System bleibt, und denen eine Alternative geboten werden muss (vgl. von Tetzchner/Martinson, 2000, S. 79ff).

Für all diese Kinder ist der Einsatz von Unterstützter Kommunikation sinnvoll. „Unterstützte Kommunikation ist die Bezeichnung für das international etablierte Fachgebiet AAC (Augmentative and Alternative Communication), das sich die Verbesserung der kommunikativen Möglichkeiten von Menschen mit schwer verständlicher bzw. fehlender Lautsprache zum Ziel gesetzt hat. Wie es der internationale Terminus verdeutlicht, geht es bei diesem Ansatz um Kommunikationsformen, die unzureichende Lautsprache ergänzen (augmentative communication) bzw. ersetzen (alternative communication)" (Braun, 2005, S. 01.003.001).
Sie wird voraussetzungslos angewendet, d.h. jedes Kind, das (noch) nicht lautsprachlich kommuniziert, kann von UK profitieren. Ihr Einsatz sollte früh beginnen, um die Zeit zu nutzen, in der Kinder besonders sensibel für den Erwerb von Sprache sind und sie spielerisch und leicht erlernen. Sie kann und will nicht die logopädische Behandlung ersetzen, in vielen Fällen bereitet sie diese vor, weil die Kinder durch UK bereits lernen, dass Kommunikation Spaß macht, dass man damit etwas erreichen und mit anderen interagieren kann. Darüber hinaus werden erste grundlegende Regeln der kommunikativen Interaktion gelernt: einen Dialog zu initiieren, aufrechtzuerhalten, Rückmeldung zu geben, Antlitzgerichtetheit usw.

Die Formen der UK unterscheiden sich in körpereigenen Kommunikationsformen und Kommunikationshilfen, die wiederum in nichtelektronische und elektronische Hilfen unterteilt werden.

Die erste körpereigene Kommunikationsform, über die ausnahmslos alle Säuglinge verfügen, ist die Körpersprache. Sie wird von den Eltern interpretiert. Auch behinderte Säuglinge verfügen über Körpersprache: Atemrhythmus, Anspannung, Vokalisationen, Blickrichtung und Körperhaltung geben Hinweise über die Befindlichkeit oder Wünsche eines Kindes. Manchmal sind diese Hinweise aber schwerer zu entschlüsseln, weil sie nicht denen der nicht behinderten Kinder entsprechen. So fällt es zum Beispiel manchen behinderten Kindern schwer, den Kopf zu den Eltern zu drehen oder Blickkontakt aufzunehmen und zu halten, viel- 
leicht verändert sich aber ihre Körperspannung o. Ä. In diesen Fällen ist es Aufgabe der UK diese ersten Zeichen aufzuspüren, zu entschlüsseln, zu erklären und Eltern zu ermuntern, darauf zuverlässig zu reagieren.

Eine Form der Körpersprache, die es ermöglicht, sich auch über Dinge auszutauschen, die sich nicht in Sichtweite befinden, sind die Gebärden. Die Ausführung von Gebärden ist motorisch weniger anspruchsvoll als die präzise Artikulation von Lauten, außerdem haben Gebärden oft mehr Ähnlichkeit mit dem Bezeichneten als Worte (z.B. das Wort "trinken" hat keine Ähnlichkeit mit dem Vorgang des Trinkens, wohl aber die allgemein übliche Gebärde dafür) und im Gegensatz zur Lautsprache kann ein Erziehender direkt in den Lernvorgang eingreifen, indem die Hände des Kindes geführt werden. Bei einigen Behinderungsarten fällt es Kindern viel leichter auf diese visuellen Reize zu reagieren als auf auditive Reize. Das gilt insbesondere für Kinder mit DownSyndrom, für die deshalb die Gebärden unterstützte Kommunikation (GuK) entwickelt wurde. Wenn Kinder hören können, sollten die Gebärden immer gleichzeitig mit Lautsprache angeboten werden. In der Regel führt das gleichzeitige Gebärden der Erziehenden dazu, dass sie langsamer und deutlicher sprechen.

Nicht elektronische Kommunikationshilfen können greif- und tastbare Gegenstände sein, die dem Kind signalisieren, worüber im Augenblick gesprochen wird oder was von ihm erwartet wird. Sie sind besonders für Kinder geeignet, die visuelle Beeinträchtigungen haben. So kann eine CD für Musik stehen, ein Waschlappen kündigt die Körperpflege an usw. Allerdings ist es schwierig, immer geeignete Gegenstände zu finden und diese auch so zu lagern, dass diese schnell verfügbar sind.

Günstiger ist der Einsatz von Bildern, Piktogrammen und Fotos. Diese zweidimensionalen Zeichen haben mehrere Vorteile. Ihre Grö-
Be, Komplexität und Farbigkeit kann auf die visuellen Fähigkeiten des jeweiligen Kindes angepasst werden. Es steht eine Vielzahl von Symbolsammlungen zur Verfügung. Sie sind nicht flüchtig wie die Laut- oder Gebärdensprache. Sie können fast überall angebracht werden: auf Kommunikationstafeln, in Kommunikationsbüchern, auf Tischsets, auf Schürzen, an der Wand, am Schrank usw. Sie lassen sich beschriften, sodass auch Personen, die die Bedeutung nicht direkt erkennen, diese nachlesen können. Sie müssen lediglich wiedererkannt werden, im Gegensatz zu Gebärden, die man erinnern muss, wenn man sie gebrauchen möchte. Sie sind leicht herzustellen, gut transportabel und preiswert.

Die letzte große Gruppe der Kommunikationshilfen sind die elektronischen Hilfen. Hier spricht man von kleinen oder einfachen Hilfen und komplexen elektronischen Hilfsmitteln (vgl. Breul, 2011). Die einfachsten sind „sprechende Tasten". Auf ihnen kann schnell und einfach eine Aussage aufgenommen und durch Tastendruck ausgelöst werden. Zunächst ist es dabei nicht unbedingt erforderlich, dass das Kind versteht, was es mithilfe der Taste mitteilt, sondern lediglich, dass es erfährt, dass Sprache zu einer positiven Reaktion aus der Umwelt führt. Anspruchsvollere Hilfen mit statischem Display geben je nach Modell die Möglichkeit, bis zu etwa 30 Aussagen zu machen. Um gezielt etwas Bestimmtes zu sagen, ist es notwendig, die Tasten zu bebildern oder zu beschriften, damit man erkennen kann, welche Aussage unter welcher Taste verborgen ist. Die Äußerungen müssen bei einfachen elektronischen Kommunikationshilfen vorher aufgesprochen werden. Wenn man die Aussagen ändern will (zum Beispiel beim Wechsel vom Kindergarten nach Hause), muss ein anderes Deckblatt auf die Oberfläche gelegt werden.

Bei den komplexen Kommunikationshilfen mit dynamischem Display gelangt man durch Auswahl eines Symbols in eine Unterkategorie, in 
der weitere Symbole angeboten werden. Zum Beispiel können durch die Aktivierung des Symbols für Essen verschiedene Mahlzeiten angeboten werden: Frühstück, Mittag, auswärts Essen. Unter auswärts Essen könnte z. B. ein Eis von der Eisdiele oder ein Fastfood-Restaurant angeboten sein. Es wird häufig ein Wortschatz mitgeliefert, man muss dann nicht vorher selber alles aufsprechen. Es gibt verschiedene Methoden, um mit möglichst wenig Tasten möglichst Vieles genau ausdrücken zu können. Die Vorteile der elektronischen Hilfsmittel sind, dass man sich mit ihrer Hilfe auch bemerkbar machen kann, wenn gerade keiner guckt; sie sind sozial gut akzeptiert; auch Personen, die nicht lesen können oder keine Gebärden kennen, verstehen sie. Ihr größter Vorteil ist, dass die Aktivierung der Schalter an jede Art der körperlichen Beeinträchtigung angepasst werden kann, sodass sie ggf. auch durch Blicke ausgelöst werden können.

Im Gegensatz zum Erwerb einer Fremdsprache geht es beim Erlernen von UK nicht darum, eine der Formen perfekt zu beherrschen, sondern die Formen werden miteinander kombiniert, sodass das jeweilige Kind die ganz individuelle Kommunikationsform einsetzen kann, die es ihm und seinen Bezugspersonen erlaubt, sich effektiv, effizient und angenehm miteinander zu verständigen. Bei der Auswahl der geeigneten Kommunikationsformen sollten die Eltern und wenn möglich das Kind einbezogen werden.

Im Zusammenhang mit UK wird immer wieder die wichtige Frage gestellt, ob der Einsatz von UK nicht dazu führen kann, dass die Kinder „faul“ werden, weil sie sich ja nicht mehr anzustrengen brauchen, um verstanden zu werden und dadurch in ihrer Lautsprachentwicklung behindert würden. Sowohl wissenschaftliche Untersuchungen als auch Berichte aus der Praxis zeigen, dass Kinder, sobald sie die Fähigkeit entwickeln, sich lautsprachlich zu verständigen, die UK zugunsten der Lautsprache aufgeben; denn dies ist die Sprache, in der sich ihre Bezugspersonen miteinander verständigen. Im Gegenteil kommen viele Kinder über den Weg der UK in die Lautsprache (Wilken, 2006; Kaufmann, 2006).

\section{Literatur}

Braun, U. (2005): Was ist Unterstützte Kommunikation. In: von Loeper Literaturverlag; ISAAC, Gesellschaft für Unterstützte Kommunikation (Hrsg.): Handbuch der Unterstützten Kommunikation. Band 1. Karlsruhe: von Loeper. S. 01.003.001-01.005.001

Breul, W. (2011): Elektronische Kommunikationshilfen - Ein Überblick. In: von Loeper Literaturverlag; ISAAC, Gesellschaft für Unterstützte Kommunikation (Hrsg.): Handbuch der Unterstützten Kommunikation. Band 1. Karlsruhe: Von Loeper. S. 04.005.001-04.011.001

Kaufmann, U. (2006): Sprachentwicklung unterstützt kommunizierender Kinder. Eine qualitative Pilotstudie mit 10 Kindern im Alter von 3-6 Jahren. Diss. Universität zu Köln Von Tetzchner, S., Martinsen, H. (2000): Einführung in die Unterstützte Kommunikation. Heidelberg: Universitätsverlag C. Winter Wilken, E. (2006): Präverbale sprachliche Förderung und Gebärdenunterstützte Kommunikation in der Frühförderung. In: Wilken, E. (Hrsg.): Unterstützte Kommunikation. Eine Einführung in Theorie und Praxis. 2. Auflage. Stuttgart: Kohlhammer 